\title{
A SURVEY OF NEUTRAL HYDROGEN AT HIGH GALACTIC LATITUDES
}

\author{
W. C. ERICKSON, H. L. HELFER, AND H. E. TATEL* \\ Department of Terrestrial Magnetism, Carnegie Institution, \\ Washington, D.C., U.S.A.
}

\section{INTRODUCTION}

Approximately 1000 observations of neutral hydrogen have been obtained with the 54-channel $\mathrm{H}$-line receiver and the Würzburg antenna of the Carnegie Institution of Washington. H-line profiles have been observed at 10-degree intervals along the \pm 20 -, \pm 30 -, and \pm 40 -degree parallels of galactic latitude; at 20-degree intervals along the \pm 50 - and \pm 60 -degree parallels; at 40-degree intervals along the \pm 70 - and \pm 80 -degree parallels and at the poles. Approximately two dozen observations have been taken at points near the galactic plane in order to correlate these observations with the Leiden survey [1]. The beamwidth of the Würzburg antenna was about 2 degrees. The observations were taken in two series, one series during the summer of 1957, and the other series during 1958 January. The video frequency bandwidth of the receiver is $12 \mathrm{kc} / \mathrm{s}$. The profiles consist of averages of from two to six scans with integration times from 4.8 to 7.5 minutes.

\section{DISCUSSION OF THE DATA}

In Fig. 1 we show a typical example of the original data obtained in this work. This point was observed in $1957 \mathrm{July,}$ and again in 1958 January.

In discussing these data it should be emphasized that the gas observed at high galactic latitudes is almost certainly local. Even at $|b|=20$ degrees we do not expect to observe gas at distances greater than 1 kiloparsec.

We first discuss the results that are obvious upon a casual inspection of the data. In Fig. 2 plots are shown of our results. We plot isotherms of antenna temperature against galactic longitude as the abscissa, and radial velocity as the ordinate. The first conclusion is that the $\mathrm{H}$-line signals are generally more intense in the southern galactic hemisphere than in the northern hemisphere. It appears that gas in the northern hemisphere avoids the longitude range $l=30$ to 90 to 180 degrees. In the southern hemisphere the distribution is probably more uniform and generally more intense. However, there would appear to be a preference for the same longitude region which is avoided in the northern hemisphere. Another very interesting effect that we observed is that the mean radial velocity shifts progressively negative by 5 to $10 \mathrm{~km} /$ second from $|b|=20$ degrees to $|b|=70$ or 80 degrees. These

* Deceased 1957 November 15. 


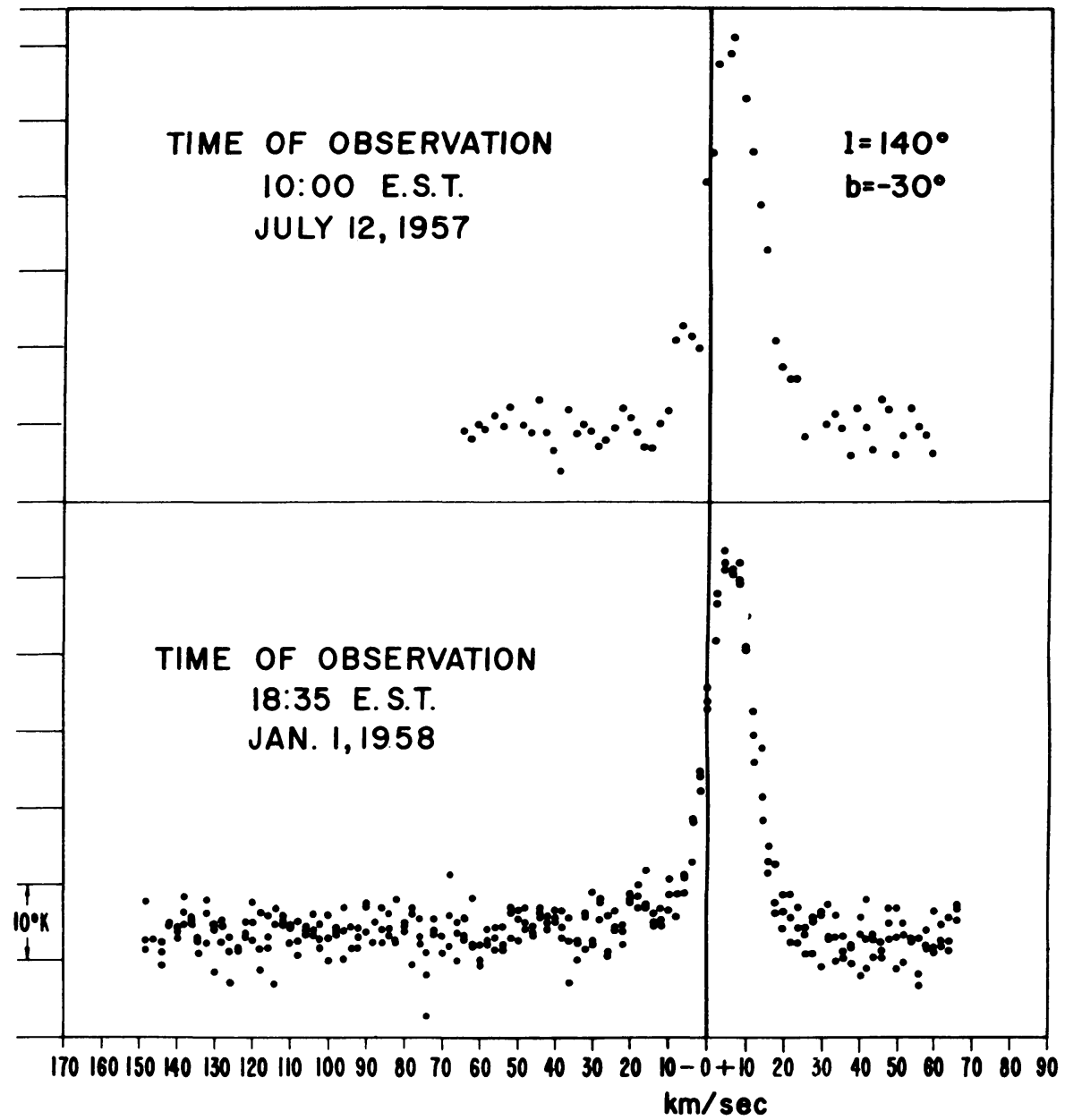

FIG. 1. Examples of the original data obtained at galactic coordinates $l=140^{\circ}, b=-30^{\circ}$.

TABLE I

Average H-Line Areas and Velocities

$\begin{array}{cccccc}b & \begin{array}{c}\text { Area } \\ { }^{\circ} \mathrm{K} \mathrm{km} / \mathrm{sec}\end{array} & \begin{array}{c}\text { Average } \\ \text { Velocity } \\ \mathrm{km} / \mathrm{sec}\end{array} & b & \begin{array}{c}\text { Area } \\ { }^{\circ} \mathrm{K} \mathrm{km} / \mathrm{sec}\end{array} & \begin{array}{c}\text { Average } \\ \text { Velocity } \\ \mathrm{km} / \mathrm{sec}\end{array} \\ 20 & 430 & 0.0 & -20 & 480 & 3.8 \\ 30 & 300 & -2.2 & -30 & 370 & 2.3 \\ 40 & 210 & -1.9 & -40 & 370 & 0.6 \\ 50 & 100 & -4.0 & -50 & 290 & -3.7 \\ 60 & 70 & -6.1 & -60 & 190 & -6.0 \\ 70 & 30 & -3.0 & -70 & 180 & -5.2 \\ 80 & 40 & -2.4 & -80 & 160 & -6.8\end{array}$



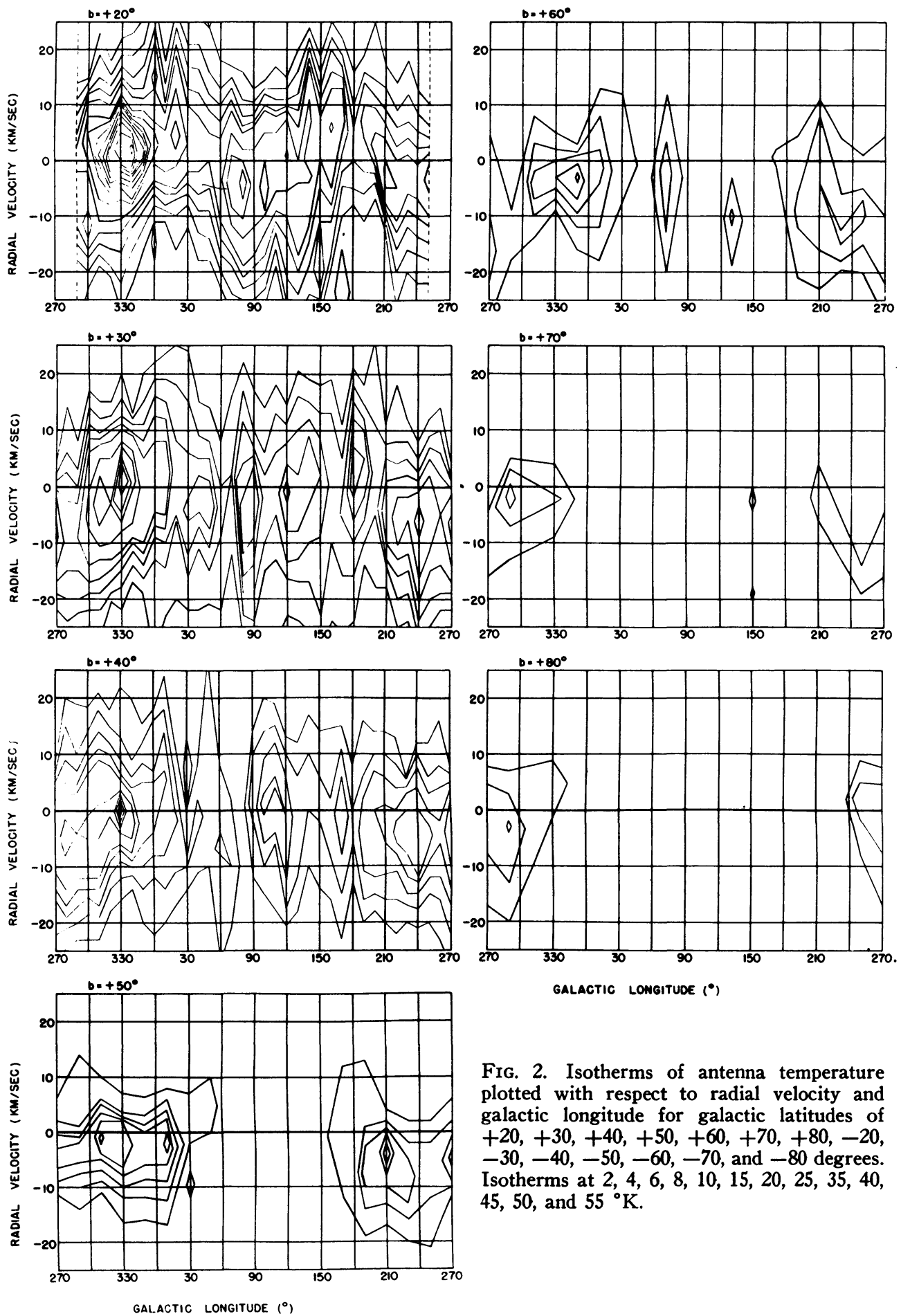

gaLACTIC LOMGITUDE ( $\left(^{\circ}\right.$

FIG. 2. Isotherms of antenna temperature plotted with respect to radial velocity and galactic longitude for galactic latitudes of $+20,+30,+40,+50,+60,+70,+80,-20$, $-30,-40,-50,-60,-70$, and -80 degrees. Isotherms at $2,4,6,8,10,15,20,25,35,40$, 45,50 , and $55^{\circ} \mathrm{K}$. 

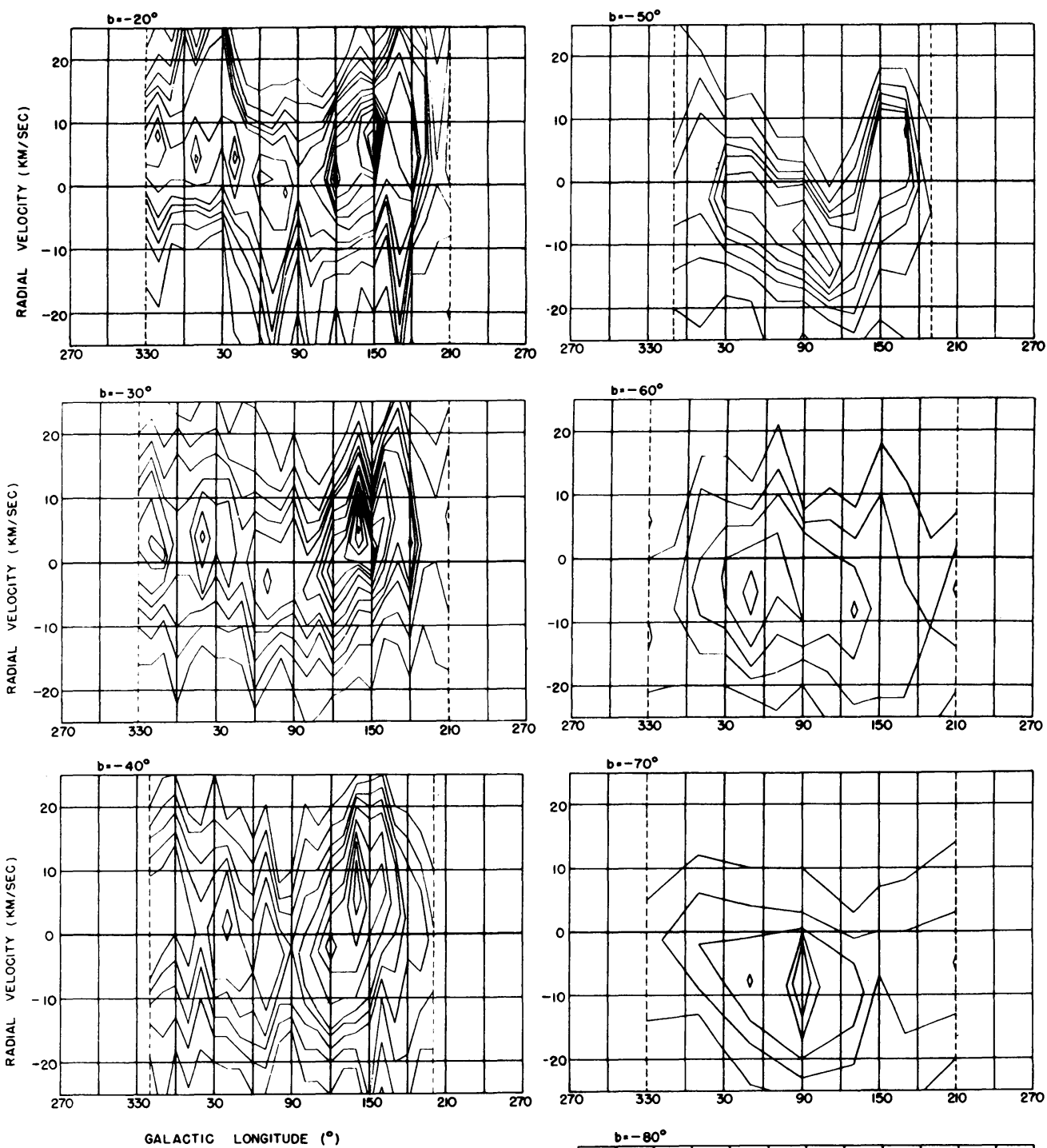

GALACTIC LONGITUDE $\left({ }^{\circ}\right)$

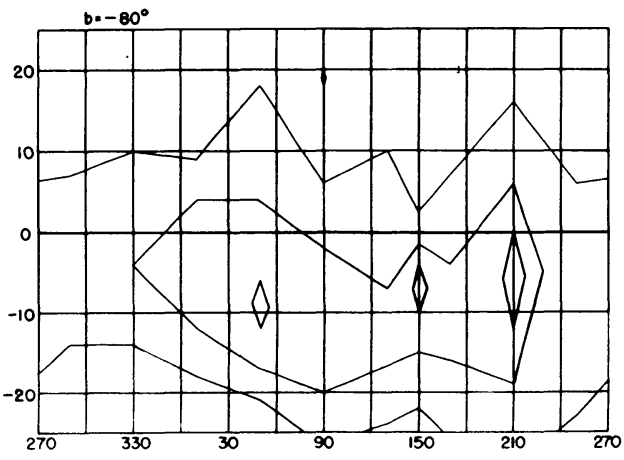

GALACTIC LONGITUDE $\left({ }^{\circ}\right)$ 
effects are illustrated in Table I where the average areas under the H-line profiles and the average velocities are shown for the northern and southern latitudes.

We also find an asymmetry in radial velocity between $b=+20$ and $b=-20$ degrees. That is, at $b=+20$ degrees the radial velocity of the gas appears to be roughly 0 to $-1 \mathrm{~km} / \mathrm{second}$, depending, upon how one chooses to weight the data. At $b=-20$ degrees, however, the average velocity is roughly $+4 \mathrm{~km} /$ second. This asymmetry is also observable at $b= \pm 30$ and \pm 40 degrees although it is smaller in amplitude and superimposed upon the general shift toward the negative velocities indicated above.

\section{PRELIMINARY INTERPRETATION OF THE DATA}

We have devoted some thought to possible interpretations of these data. However, it should be emphasized that the following comments are of a somewhat speculative nature. The motion of the gas is generally circular with some complex noncircular components. At $|b|=20$ degrees there are two main concentrations of hydrogen; one is at +20 degrees and toward the galactic center, the other is diametrically opposite in the sky, at -20 degrees toward the anti-center. The Harvard group has suggested that this may be associated with the Gould's Belt phenomenon. Both of these concentrations, however, have a mean radial velocity of about $+4 \mathrm{~km} / \mathrm{second}$. Therefore a $\mathrm{K}$-term may be needed to explain these velocities. There are other indications of a K-term. One is the fact that the mean velocity in the $b=-20$ degrees survey is generally positive even in the negative quadrant from $l=60$ to 150 degrees. This fact also indicates the presence of a K-term. In the $b=+20$ degrees survey the gas from $l=190$ to 240 degrees has a generally negative radial velocity, whereas according to a model of strictly circular galactic motion one would expect this gas to possess positive radial velocity. One sees a somewhat sinusoidal character to the plots of the $|b|=20-, 30$-, and 40-degree data suggesting circular motion. However, this sine wave is often somewhat displaced with respect to zero velocity, and even with respect to such a displaced zero line the gas does not "zero" precisely at the four nodes; i.e., $l=327,57,147$, and 237 degrees, but the median line generally passes through zero within 20 degrees in longitude of these nodes. We have attempted to fit these data by assuming a model of systematic peculiar motion in the solar neighborhood (such as streaming along galactic arms) superimposed upon circular motion. These attempts, however, have not been particularly successful, Our primary conclusion is that the motion is complex, not simply explicable through a $\mathrm{K}$-term or a streaming motion.

If one were situated near a galactic arm and were to observe it on a survey such as this, one might expect to see the arm disappear in the distance in directions roughly 180 degrees apart. Such an arm could not be traced for very large distances because when one observes it at high latitudes the line of sight rapidly leaves the galactic plane. On the plots shown in Fig. 2 one might expect the regions where arms are disappearing in the distance to 
appear with high radial velocities. One of us (H.L.H.) has attempted to interpret these data on the basis that an arm disappears in the distance at $l=180$ degrees, extends through 150 and 90 degrees, and disappears again at $l=20$ degrees. Another arm may extend from $l=200$ degrees through 240 and 300 degrees, and disappear at $l=0$ degrees. The gas appears to be spread considerably in distance when one observes in a direction perpendicular to such arms as in the region of $l=60$ degrees. One very difficult feature to interpret is the large velocity spread near the anti-center.

Fig. 3 displays the total areas under the hydrogen-line profiles. The absence of gas at high northern latitudes in the region $l=30$ to 90 to 180 degrees is easily visible. From 180 through 270 to 30 degrees there is considerable gas above the plane. Observations from the southern hemisphere are needed to complete the survey. However, from the form of our contours and from the Christiansen-Hindman survey [2], one would assume that there may not be much hydrogen below the plane in the latter longitude region. This general distribution may be summarized in the following way: consider the meridian plane passing through $l=90$ and $l=270$ degrees. Strong signals are observed in the quadrant between $l=270, b=0$, and $b=+90$ degrees, but weak signals between $b=+90, l=90$, and $b=0$ degrees. We find strong signals again in the quadrant between $l=90, b=0$, and $b=-90$ degrees, and weak

\section{TOTAL AMOUNT OF NEUTRAL HYDROGEN}

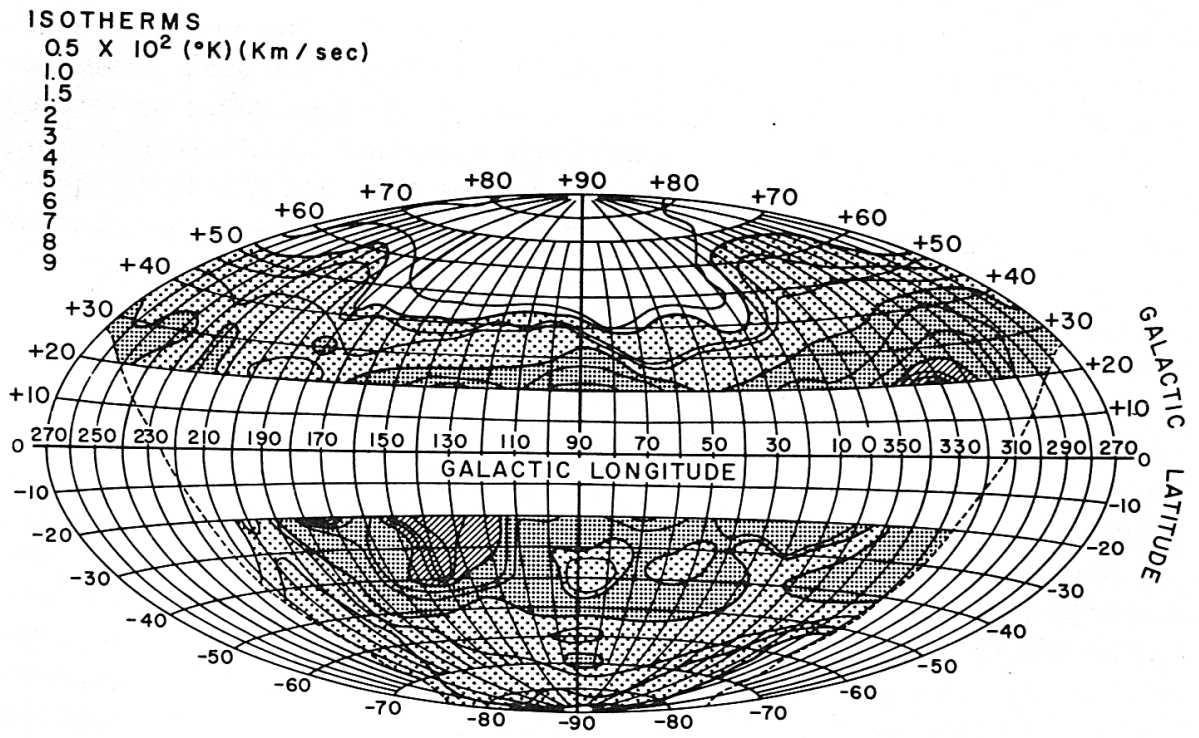

Fy. 3. A map of the variation of total areas under the H-line profiles. These areas are proportional to the total number of neutral hydrogen atoms in the line of sight. To obtain the number of atoms per $\mathrm{cm}^{2}$ the units of $\left({ }^{\circ} \mathrm{K}\right)(\mathrm{km} / \mathrm{second})$ should be multiplied by roughly $2 \times 10^{18}$. 
signals in the fourth quadrant. We might suggest that this plane is roughly perpendicular to the spiral arms in the solar neighborhood. Then the data may be interpreted as showing either that the sun is situated between two spiral arms in the quadrants of strong signals or that the sun is imbedded somewhere above the center of a single spiral arm of elliptical cross section with the major axis of the ellipse near the line $l=270, b=+45$ degrees.

The authors wish to express their appreciation to the Convair Scientific Research Laboratory for financial support and technical assistance in the reduction of these data. We particularly wish to acknowledge the work of Mr. Delano Ball who performed many of the more laborious tasks in connection with these reductions. The untimely death of Dr. Howard E. Tatel is to be acknowledged. His death is a severe loss to our science, and he is keenly missed by his friends and associates.

\section{REFERENCES}

[1] Muller, C. A., et al. B.A.N. 475, 151, 1957.

[2] Christiansen, W. N., and Hindman, J. V. Aust. J. Sci. Res. A 5, 437, 1951.

\section{Discussion}

Bok: It is a cause of deep regret to all of us here that one of the authors of this paper, Dr. Howard Tatel, is no longer with us. His untimely death means a great loss for the field of radio astronomy. We shall remember Howard Tatel as the man who first succeeded in constructing operational multi-channel $21-\mathrm{cm}$ equipment. Tatel made important contributions toward the design of the 140-foot radio telescope for the U.S. National Radio Astronomy Observatory at Green Bank, and the telescope to be mounted there will be in many ways a lasting memorial to Howard Tatel's pioneer thinking.

Westerhout: The symmetry around $l=90$ degrees in the distribution of neutral $\mathrm{H}$ at high latitudes seems to be spurious, since $l=90$ degrees is about in the center of the region investigated.

Erickson: We did not center our observations on $l=90$ degrees particularly. This meridian has no particular significance with respect to our observing program except for the fact that the north celestial pole lies on this meridian, and that the region in the southern hemisphere that is unobservable from Washington is centered on the $l=270$ degree meridian. There is no instrumental reason why the $l=90$ degree meridian should have any particular significance. The only reason I chose to plot my graphs with this meridian in the center was that this gives roughly equal prominence to the two dominant features lying near the galactic center and anti-center.

Blaauw : Will your available observations permit the study of the long tails at high velocities $\pm 100 \mathrm{~km} / \mathrm{second}$ ?

Erickson: Our observations extend over a frequency range wide enough 
that under ideal conditions we should have been able to observe high-velocity wings on the profiles, if they exist. However, during these observations our base lines were unsteady, and would often change by 2 or $3{ }^{\circ} \mathrm{K}$ as dew or frost formed on our antenna feed. These fluctuations made it impossible to confirm or dispute definitely the Dutch observations of such wings. The situation is improving, however, and the Carnegie group plans to make such observations in the future.

Schmidt: I understand that the smoothness of the profiles at high latitude cannot be reconciled with a model of small discrete clouds in interstellar space. Is this right?

Erickson: I have the impression from studying these data that there exists considerable structure between the observed points. There are fairly large, and not particularly smooth amplitude fluctuations from point to point. A finer grid of observations would be needed to settle the question of whether or not these are due to individual, distinct clouds. However, the variation in the velocities of the observed peaks is surprisingly smooth from point to point. This may be irreconcilable with a picture of individual interstellar clouds possessing distinct random velocities.

van de Hulst: With reference to Dr. Schmidt's question, it would seem that the angular size of the details observed at high latitudes is not sufficiently different from what would be expected on the basis of an average distance of 100 parsecs and a diameter of 10 to 50 parsecs for a typical cloud.

Erickson: Yes, for the amplitude variations this is roughly the case. 